\title{
Editorial
}

Dermatology

\section{Linear Immunoglobulin A Bullous Dermatosis: Need for an Agreement on Diagnostic Criteria}

\author{
Emiliano Antiga Marzia Caproni Paolo Fabbri \\ Department of Medical and Surgical Critical Care, Section of Dermatology, University of Florence, Florence, Italy
}

\section{Key Words}

Linear immunoglobulin A bullous dermatosis · Bullous

pemphigoid · Diagnostic criteria

\begin{abstract}
Linear immunoglobulin A bullous dermatosis (LABD) is a rare, heterogeneous, autoimmune blistering disorder. Although the main characteristics of the disease seem to be well identified, international accepted diagnostic criteria are lacking. Several authors suggested their own criteria, but they are often not complete or even in contrast with clinical and immunopathological findings from the literature, while others are too selective. In this review, the current views and the problems with the definition of reliable diagnostic criteria for $L A B D$ will be discussed.

๑) 2013 S. Karger AG, Basel
\end{abstract}

\section{Introduction}

Linear immunoglobulin A (IgA) bullous dermatosis (LABD) is a rare, heterogeneous, acquired, subepithelial, autoimmune blistering disorder. The etiology of LABD is not fully understood; however, cases are reported in as- sociation with drugs, infections as well as malignancies. The main features of the disease are well known and have been recently reviewed in detail [1-3]. LABD usually presents with vesicles, blisters and/or erosions involving the skin and - frequently - the mucous membranes [1]. Histopathologically, LABD is characterized by subepidermal blister formation with a predominantly neutrophilic infiltrate in the lesional skin, features that can be observed in other subepidermal blistering diseases such as bullous pemphigoid (BP) and epidermolysis bullosa acquisita (EBA). However, the most important feature of LABD is the presence of continuous linear IgA deposits at the basement membrane zone (BMZ) on direct immunofluorescence microscopy of perilesional skin; such finding should not be confused with the deposition of granular IgA at the tips of the dermal papillae or along the $\mathrm{BMZ}$ that are typical of dermatitis herpetiformis [4]. Another important immunopathological finding is the presence of circulating IgA autoantibodies directed against heterogeneous BMZ antigens (table 1). Finally, LABD typically responds to dapsone, although other autoimmune bullous diseases also variably do $[1,2]$.

There are two main variants of LABD: LABD of the adult and of the child, often called chronic bullous disease of childhood. These two forms show some differences in

\section{KARGER}

E-Mail karger@karger.com

www.karger.com/drm
(C) 2013 S. Karger AG, Basel

$1018-8665 / 13 / 2264-0329 \$ 38.00 / 0$
Emiliano Antiga, MD, $\mathrm{PhD}$

Department of Medical and Surgical Critical Care Section of Dermatology, University of Florence

Piazza Indipendenza, 104, IT-50129 Florence (Italy)

E-Mail emiliano.antiga@ unifi.it 
Table 1. Antigens targeted in LABD

\begin{tabular}{ll}
\hline Molecular weight, $\mathrm{kDa}$ & Name (if available) \\
\hline 97 & LABD97 \\
100 & \\
$110-120$ & LAD-1 \\
120 & \\
145 & \\
$160-180$ & BPAg2 \\
180 & laminin- $\gamma 1$ chain \\
200 & \\
220 & BPAg1 \\
230 & \\
255 & LAD285 \\
285 & collagen VII \\
\hline
\end{tabular}

their clinical presentation (including a higher responsiveness to dapsone and a higher percentage of self-resolution of the childhood variant) $[1,5]$.

\section{Diagnostic Criteria for LABD Are Lacking}

Although the main characteristics of the disease seem to be well identified, no diagnostic guidelines have been published yet and international accepted diagnostic criteria are lacking. Several authors have suggested their own criteria.

For example, Sheridan et al. [6] proposed that prominent linear IgA deposition at the BMZ, irrespective of the presence of circulating IgG, is consistent with a diagnosis of $\mathrm{LABD}$, which justifies the introduction of dapsone as firstline treatment. The presence of acquired bullous disease and solely linear IgA at the BMZ in clinically uninvolved skin were the diagnostic criteria used by Lally et al. [7].

Bertram et al. [8], in a prospective analysis of the incidence of autoimmune bullous disorders in a region of Germany, made the diagnosis on the basis of the following criteria without any further detail and specification: blisters/erosions not predominantly on mucous membranes; IgA > IgG along the BMZ on direct immunofluorescence microscopy; IgA > IgG on the blister roof on salt split skin; IgA reactivity to BP180 fragments on immunoblotting.

Finally, in a recently published study on the presence of IgA in the pemphigoids, Horváth et al. [9] based the diagnosis of LABD on more restrictive criteria, including the presence of subepidermal blisters with a neutrophilrich inflammatory infiltrate, of IgA deposits at the dermo-epidermal BMZ of perilesional skin by direct immunofluorescence microscopy, and of serum IgA binding to the epidermal site of $1 \mathrm{~mol} / \mathrm{l} \mathrm{NaCl}$-separated normal human skin by indirect immunofluorescence.

\section{Problems with the Definition of Reliable Diagnostic Criteria for LABD}

Given these reports, it seems clear that no accepted diagnostic criteria of LABD are currently available. Furthermore, several of the criteria reported above are at least not complete or even in contrast with clinical and immunopathological findings from the literature, while others are too selective.

Regarding the clinical manifestations, while some authors considered only the presence of blisters and/or erosions a valid clinical diagnostic criterion $[7,8]$, some cases of 'atypical' presentation of LABD are reported, including the novel prurigo-like variant [10]. Moreover, the clinical features of LABD are very heterogeneous and are often not distinguishable from those of other autoimmune bullous diseases, such as BP.

Histopathological findings are considered by some authors [9], but are not included in other sets of diagnostic criteria; however, since the histopathology of LABD is very similar to that of other pemphigoids, its usefulness in the diagnosis of the disease is very relative.

More problems arise with respect to the immunopathological features, which should be the most critical to differentiate LABD from other autoimmune subepidermal blistering diseases.

Firstly, although the linear IgA deposit at the BMZ is the finding that gives the name to the disease, it is not clear yet whether a diagnosis of LABD can be made only in the presence of solely linear IgA deposits [7], or even in the case of linear deposits of multiple immunoglobulin, but with IgA predominance $[6,8]$. Moreover, to define predominance of IgA based on immunofluorescence staining intensity is very complicated, since the staining intensity critically depends on the experimental conditions, including dilution and type of the second step antibodies used for detection of the tissue-bound deposits. This question becomes more important considering that linear IgA deposits have been reported in several cases of subepidermal blistering diseases other than LABD, including BP [11], mucous membrane pemphigoid (MMP) [12], pemphigoid gestationis [13] and EBA [14]. 
Another important question about the immunopathological features of LABD concerns the presence of circulating IgA antibodies against BMZ antigens. Horváth et al. [9] found them in 6 out of 6 patients by immunoblot analysis in their study, and included the presence of serum IgA binding to the epidermal site of $1 \mathrm{~mol} / \mathrm{l} \mathrm{NaCl}-$ separated normal human skin by indirect immunofluorescence as a compulsory diagnostic criterion of LABD in their case series. However, although immunoblotting is a sensitive method to detect autoantibodies, it is usually positive in less than $80 \%$ of patients $[7,15]$ and is not used routinely because it is time-consuming and costly and not available in the majority of laboratories. Moreover, other authors reported lower detection rates of circulating antiBMZ IgA in patients with LABD than Horváth et al. [9]. In particular, indirect immunofluorescence on human skin as a substrate is positive for IgA in about $30 \%$ of patients $[6,16]$, while the use of $\mathrm{NaCl}$-separated skin showed a sensitivity of up to $80 \%$ in children, but less than $50 \%$ in adults [17].

Furthermore, even if IgA deposits on $\mathrm{NaCl}$-separated skin are prevalently found at the epidermal side, dermal binding LABD was reported in about $17 \%$ of patients in a recent study by Lally et al. [7] and occasionally, antibody binding to both sides of the split is observed $[1,16]$. Thus, it is clear that there should first be a consensus as what should be considered the reactivity and staining pattern typical and characteristic for LABD.

On the other hand, circulating IgA anti-BMZ antibodies are often observed in the other subepidermal blistering diseases $[9,18]$, and circulating IgG anti-BMZ anti- bodies represent a frequent finding in patients with LABD [19-21]. Thus, the search for circulating IgA antibodies in LABD seems to be neither sensitive nor specific for the diagnosis of the disease.

Another debating issue involves the differential diagnosis between LABD with mucosal involvement, MMP and EBA, in which IgA deposits may also be found, raising the problem of the clinical heterogeneity and wide spectrum of presentations of LABD.

\section{Conclusions}

Taking into account all the remarks reported above and the wide heterogeneity of the disease, an assessment of worldwide accepted diagnostic criteria for LABD is required and a consensus for the definition of the disease is needed. In particular, several key questions should be addressed, including the following: (1) How pertinent is it to define a disease based on immunofluorescence findings? (2) Is the disease heterogeneity related to the presence of other immunobullous diseases (i.e. BP, MMP, EBA) due to antigen spreading? (3) Which is the significance of the presence not only of IgA, but also of other antibody isotypes?

\section{Disclosure Statement}

The authors declare no conflict of interest. There was no funding.

\section{References}

1 Antiga E, Torchia D, Caproni M, Fabbri P: Linear immunoglobulin A bullous dermatosis. Exp Rev Dermatol 2009;4:495-508.

2 Venning VA: Linear IgA disease: clinical presentation, diagnosis, and pathogenesis. Dermatol Clin 2011;29:453-458.

3 Kneisel A, Hertl M: Autoimmune bullous skin diseases. Part 1: Clinical manifestations. J Dtsch Dermatol Ges 2011;9:844-856.

4 Caproni M, Antiga E, Melani L, Fabbri P; Italian Group for Cutaneous Immunopathology: Guidelines for the diagnosis and treatment of dermatitis herpetiformis. J Eur Acad Dermatol Venereol 2009;23:633-638.

5 Egan CA, Zone JJ: Linear IgA bullous dermatosis. Int J Dermatol 1999;38:818-827.
6 Sheridan AT, Kirtschig G, Wojnarowska F: Mixed immunobullous disease: is this linear IgA disease? Australas J Dermatol 2000;41: 219-221.

7 Lally A, Chamberlain A, Allen J, Dean D, Wojnarowska F: Dermal-binding linear IgA disease: an uncommon subset of a rare immunobullous disease. Clin Exp Dermatol 2007; 32:493-498.

8 Bertram F, Bröcker EB, Zillikens D, Schmidt E: Prospective analysis of the incidence of autoimmune bullous disorders in Lower Franconia, Germany. J Dtsch Dermatol Ges 2009; 7:434-440.

9 Horváth B, Niedermeier A, Podstawa E, Müller R, Hunzelmann N, Kárpáti S, Hertl M: IgA autoantibodies in the pemphigoids and linear IgA bullous dermatosis. Exp Dermatol 2010; 19:648-653.
10 Antiga E, Bellandi S, Bianchi B, Del Bianco E, Pierini I, Cozzani E, Massi D, Fabbri P, Caproni $\mathrm{M}$ : A further case of subacute prurigolike linear IgA bullous dermatosis: growing evidence of a new subset. Int J Dermatol 2012; 51:1500-1501.

11 Kirtschig G, Wojnarowska F: IgA basement membrane zone autoantibodies in bullous pemphigoid detect epidermal antigens of $270-280,230$, and $180 \mathrm{kDa}$ molecular weight by immunoblotting. Clin Exp Dermatol 1999; 24:302-307.

12 Egan CA, Taylor TB, Meyer LJ, Petersen MJ, Zone JJ: The immunoglobulin A antibody response in clinical subsets of mucous membrane pemphigoid. Dermatology 1999;198:330-335.
Diagnostic Criteria of Linear

Immunoglobulin A Bullous Dermatosis
Dermatology 2013;226:329-332 DOI: $10.1159 / 000350818$ 
13 Shimanovich I, Skrobek C, Rose C, Nie Z, Hashimoto T, Bröcker EB, Zillikens D: Pemphigoid gestationis with predominant involvement of oral mucous membranes and IgA autoantibodies targeting the $\mathrm{C}$-terminus of BP180. J Am Acad Dermatol 2002;47:780784.

14 Letko E, Bhol K, Anzaar F, Perez VL, Ahmed AR, Foster CS: Chronic cicatrizing conjunctivitis in a patient with epidermolysis bullosa acquisita. Arch Ophthalmol 2006;124:16151618.

15 Allen J, Wojnarowska F: Linear IgA disease: the IgA and $\operatorname{IgG}$ response to dermal antigens demonstrates a chiefly IgA response to LAD285 and a dermal $180-\mathrm{kDa}$ protein. $\mathrm{Br} \mathrm{J}$ Dermatol 2003;149:1055-1058.
16 Wojnarowska F, Collier PM, Allen J, Millard PR: The localization of the target antigens and antibodies in linear IgA disease is heterogeneous, and dependent on the methods used. Br J Dermatol 1995;132:750-757.

17 Wojnarowska F, Marsden RA, Bhogal B, Black MM: Chronic bullous disease of childhood, childhood cicatricial pemphigoid, and linear IgA disease of adults. A comparative study demonstrating clinical and immunopathologic overlap. J Am Acad Dermatol 1988;19:792-805.

18 Cozzani E, Drosera M, Parodi A, Carrozzo M, Gandolfo S, Rebora A: Frequency of IgA antibodies in pemphigus, bullous pemphigoid and mucous membrane pemphigoid. Acta Derm Venereol 2004;84:381-384.
19 Georgi M, Scheckenbach C, Kromminga A, Partscht K, Messer G, Bröcker EB, Zillikens D: Mapping of epitopes on the BP180 ectodomain targeted by IgA and IgG autoantibodies in patients with the lamina lucida-type of linear IgA disease. Arch Dermatol Res 2001;293:109-114.

20 Metz BJ, Ruggeri SY, Hsu S, Reed JA, Ghohestani AS, Uitto J, Ghohestani RF: Linear IgA dermatosis with IgA and IgG autoantibodies to the $180 \mathrm{kDa}$ bullous pemphigoid antigen (BP180): evidence for a distinct subtype. Int J Dermatol 2004;43:443-446.

21 Viglizzo G, Cozzani E, Nozza P, Occella C, Parodi A: A case of linear IgA disease in a child with IgA and IgG circulating antibodies directed to BPAg2. Int J Dermatol 2007;46: 1302-1304. 